University of Wollongong

Research Online

Faculty of Engineering - Papers (Archive)

Faculty of Engineering and Information

Sciences

$1-1-2005$

\title{
A mathematical model to describe bending mechanics of polypyrrole (PPy) actuators
}

Gursel Alici

University of Wollongong, gursel@uow.edu.au

Philippe Metz

Institut Francais De Mecanique Avancee, France

Geoffrey M. Spinks

University of Wollongong, gspinks@uow.edu.au

Follow this and additional works at: https://ro.uow.edu.au/engpapers

Part of the Engineering Commons

https://ro.uow.edu.au/engpapers/66

\section{Recommended Citation}

Alici, Gursel; Metz, Philippe; and Spinks, Geoffrey M.: A mathematical model to describe bending mechanics of polypyrrole (PPy) actuators 2005.

https://ro.uow.edu.au/engpapers/66 


\section{A Mathematical Model to Describe Bending Mechanics of Polypyrrole (PPy) Actuators}

\author{
Gürsel ALICI \\ School of Mechanical, Materials \\ and Mechatronics Engineering \\ University of Wollongong \\ 2522, NSW, Australia
}

\author{
Philippe METZ \\ Institut Francais De Mecanique Avancee \\ Campus De Clermont-Ferrand \\ BP 265 F-63172 AUBIERE, Cedex
}

France

\author{
Geoffrey M. SPINKS \\ School of Mechanical, Materials \\ and Mechatronics Engineering \\ University of Wollongong \\ 2522, NSW, Australia
}

\begin{abstract}
In order to make use of conducting polymer actuators such as PPy actuators suitable for many cutting edge applications, and more importantly to provide enhanced degrees of understanding and predictability in quantifying their performance, it is needed to establish a valid mathematical model of such actuators. With this in mind, the aim of this study is to establish and experimentally validate a lumped-parameter model of strip-type PPy actuators for use in improving their displacement and force outputs. With reference to their operation principle, we draw an analogy between the thermal strain and the real strain in the PPy actuators due to the volume change to set up the mathematical model, which is a coupled structural/thermal model. The Finite Element Method (FEM) is used to solve the model. The rate of propagation of the ion migration into the PPy layers is mimicked with a temperature distribution model. Theoretical and experimental results demonstrate that the model is practical and effective enough in predicting the bending angle and bending moment outputs of the PPy actuators quite well for a range of input voltages. We claim that it is a fruitless task to include the influence of their mechanical, electrical and chemical parameters in an analytical model describing their input/output behaviour.
\end{abstract}

\section{I.INTRODUCTION}

After the production of nanoparticles and nanostructured materials, the next and most challenging phase of nanotechnology is to construct nanosystems/nanodevices. To this aim, a manipulation system is needed to manipulate and assemble microsized (hopefully nano-sized! ) components in order to build functional systems. Although many microsized objects/components have been fabricated using various techniques, a limited number of microelectromechanical devices consisting of a multiple number of these components have come into existence. The primary reason is nonexistence of a micromanipulation system to bring the components together in a mass production manner. The precision and resolution of such a system mainly depends on their mechanical structure and actuators, which are the dominant factors determining such strict operational requirements. These can be achieved by employing a mechanism that moves only by deformation, typically by utilizing flexure joints in place of conventional joints. In the micro/nano robotics literature, elastic elements such as flexures or notches in a rigid structure are known as flexure joints. We draw the same analogy and call a conducting polymer strip driving a rigid link an active flexure joint (acti- flex) as it acts as an actuator and a joint. Such a concept needs to be theoretically and experimentally evaluated before utilising it. The outcomes of this study will pave the way towards optimising the size of such active flexure joints and calculating the minimum displacement they can generate in order to make mechanisms to be dedicated to micro/nano manipulation systems [1].

Polymers derived from pyrrole, aniline or thiophene can be used as Conducting Polymer (CP) actuators or artificial muscles [2], [3]. The CP actuators based on pyrrole is known as polypyrrole (PPy) actuator. When the polymer is doped electrochemically, ions are sent inside the polymer causing volume expansion. Applying voltages as small as $1 \mathrm{~V}$ controls the volume change of the polymer in the form of expansion and contraction. The change in the volume generates a bending displacement. This follows that the electrochemical energy is converted into mechanical energy. There has been significant amount of research on CP actuators and their use in various applications in the last decade [2-9]. A comprehensive account of polymer actuators is given in [5], [7]. Zhou et al. [4] have reported on three types of polymer actuators including an ionic conducting polymer film actuator, a polyaniline actuator, and a parylene thermal actuator. They have presented their fabrication and initial performance results. Smela et al. [6] have presented the development and performance outcomes of PPy and Au bilayer conducting polymer actuators operating in electrolyte solutions. Santa et al. [10], [11] have investigated into the modeling and characterization of a muscle-like conducting polymer axial/linear actuator operating in an electrolytic cell. It is a simple lumped parameters model whose parameters are identified using the force and the change in the length data. Nemat-Nasser and Li [12], [13] have established a micromechanical bending motion model to characterize ' electrochemomechanical' response of an ionic polymermetal composite actuator consisting of Nafion (a trademark of DuPont Inc.) and platinum bilayer. The model is based on the micromechanics theory [13] that electrically unbalanced negative ions permanently fixed to the Nafion polymer generate the internal stresses. The parameters in the model are estimated using experimentally measured tip displacement. The objects of this paper are (i) to establish a 
mathematical model by drawing an analogy between the real actuation of a strip-type PPy actuator and a corresponding structure subjected to a temperature difference, (ii) to validate the model through comparing the simulation results obtained using the finite element analysis with experimental results.

This study is a part of an ongoing-project on the establishment of micro/nano manipulation systems such as grippers and planar mechanisms articulated with the fourth generation PPy actuators, which are fabricated at the Intelligent Polymer Research Institute at the University of Wollongong [2]. Conducting polymers have many promising features including low actuation voltage, operation in aquatic mediums and in air, low cost, high force output to weight ratio, very suitable to open-loop control. Their main drawback is their low speed of response and nonlinearity due to their actuation principle, which is based on mass transfer. There are a large number of variables and interrelated parameters that affect the actuation ability of PPy actuators. The complex nature of the actuation mechanisms and lack of analogous classical theories have hindered the development and conclusive experimental verification of analytical mathematical models. As the working principle of a PPy actuator involves a number mechanical, electrical and chemical parameters, we believe that it is a fruitless task to include the influence of all the parameters in an analytical model to generate a fairly accurate model. With this in mind, we develop a lumped parameter model to predict the displacement and force outputs of PPy conducting polymer actuators. The model has been solved using the FEM. The mathematical model has been validated in the voltage domain and in the thickness domain so that it can be used to optimise the geometry of PPy layers for a range of applied voltages [1].

\section{CONDUCTING POLYMER ACTUATORS}

The structure and composition of the actuator considered in this study are shown in Figure 1(a). This actuator is a composite structure consisting of five layers of three different materials; two layers of polypyrrole with a thickness of $30 \mu \mathrm{m}$ each, two layers of platinum with a thickness ranging between 10 and 100 Angstrom and one layer of porous polyvinylidine fluoride (PVDF) with a thickness of $110 \mu \mathrm{m}$.

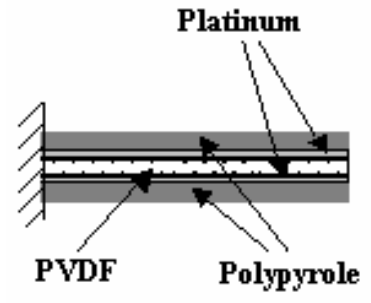

(a)

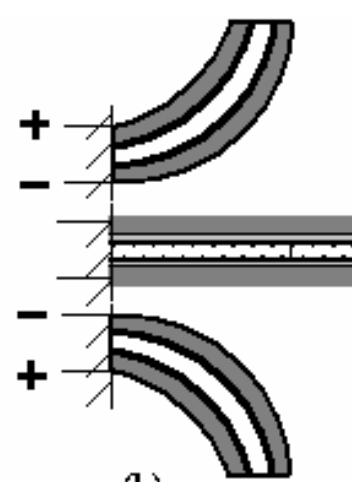

(b)
Figure 1: (a) Schematic structure of the conducting polymer actuator, and (b) Schematic representation of the bending principle.

It has been reported [7] that the Young's modulus of Polypyrrole varies between $20 \mathrm{MPa}$ and $180 \mathrm{MPa}$, depending of the value of the voltage applied. However, in this study, it is assumed that the operation voltage ranges between $[-1.0 \mathrm{~V}$; $1.0 \mathrm{~V}]$ and the Young's modulus is constant. Platinum has a Young's modulus of $171 \mathrm{GPa}$. The porous PVDF is a membrane filter Durapore ${ }^{\mathrm{TM}}$ manufactured by Millipore Pty. Ltd. As the Young's modulus of this material is not available in the literature, the modulus needs to be estimated. The approximation $E \approx E_{P}\left(\rho / \rho_{P}\right)^{n}$ where $E_{P}, \rho_{P}$ are the Young's modulus and the density of the solid PVDF gives $\mathrm{E}_{\mathrm{PVDF}}=612 \mathrm{MPa}$ [14-16]. Please note that $\mathrm{n}=1$ for most of the porous polymers.

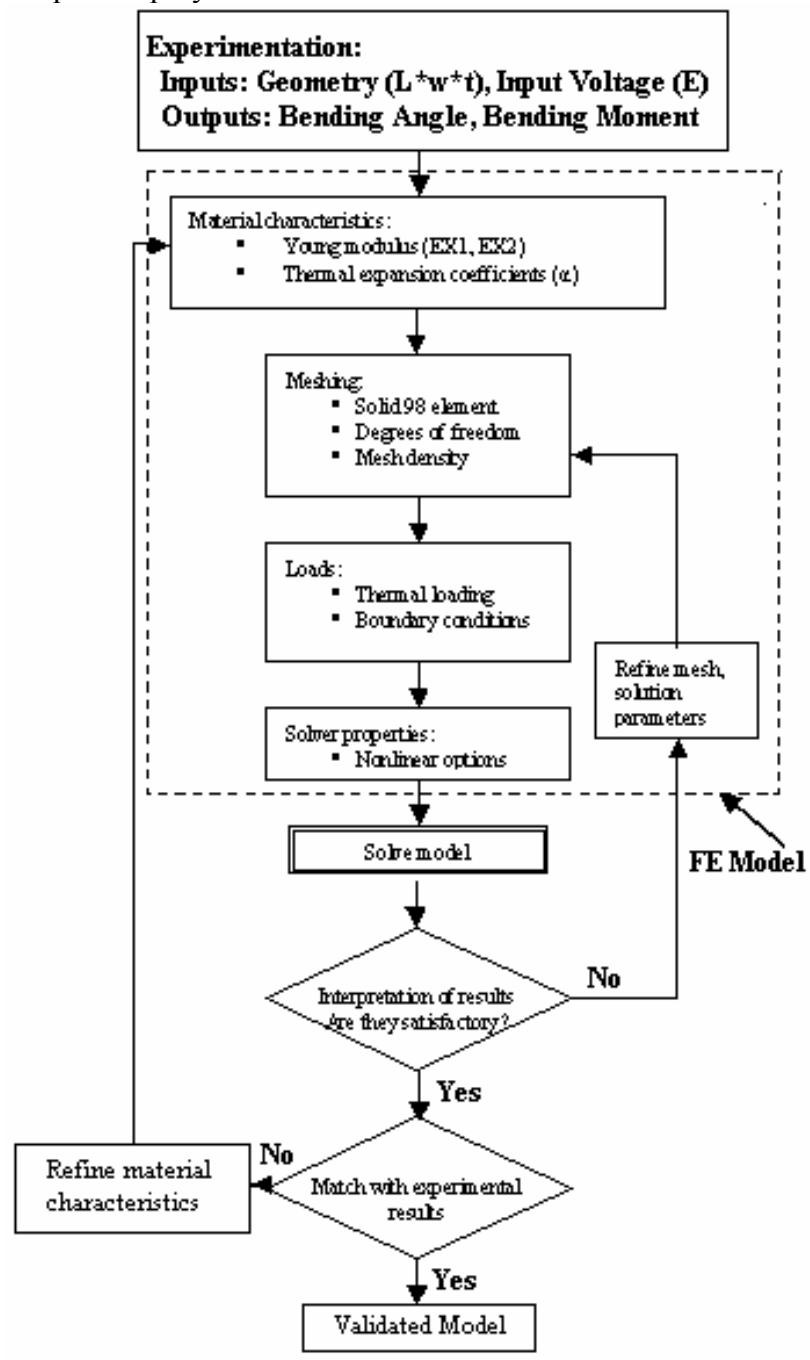

Figure 2: Flowchart for mathematical model establishment and validation.

When a current or a voltage is applied, a Red/Ox reaction occurs in the polypyrrole. So it has three states: oxidized (or doped), reductized (or de-doped) and neutral. When it is positively charged, the polypyrrole attracts an anion 
(oxidation) to balance the removal of the electron from the polymer. Following the same principle, the reduction involves the addition of electron in the polymer and the removal of the anion when it is negatively charged. These anions come from the porous PVDF, which contains an electrolyte. The doping level could be controlled by the voltage applied. The movement of ions in one hand, and the solvent molecule in the other hand, induce a volume expansion or contraction. The volume of the doped PPy layer increases and the volume of the other PPy layer decreases. Furthermore, the polymer backbone interacts electrostatically with the displaced ions. In the doped layer the polymer backbone and the anions are both negatively charged and on the contrary, in the undoped layer the cation are positively charged. The resultant electrostatic forces cause the PPy layer to expand or contract. This creates the bending motion, as seen in Figure 1(b).

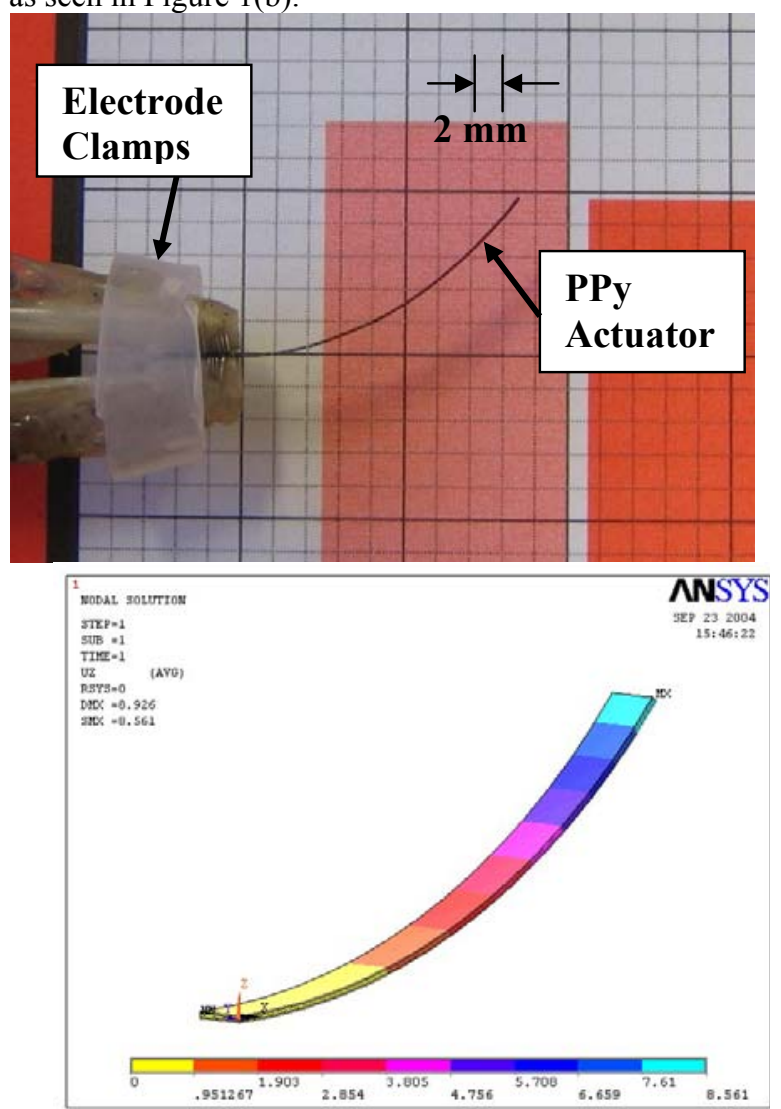

Figure 4: Reading the horizontal and vertical displacements of the actuator to calculate the angle.

\section{MODEL ESTABLISHMENT}

With reference to the operation principle of the actuator, we draw an analogy between the thermal strain and the real strain in PPy actuator due to the volume change to establish the mathematical model, which is a coupled structural/thermal mathematical model. The software ANSYS is used to solve the coupled model in order to obtain the outputs (i) the bending angle $(\theta)$, and (ii) the bending moment $(\mathrm{M})$, of the actuator. The flowchart in Figure 2 shows the steps involved in establishing and validating the mathematical model. The principle parameters used here are EX1 and EX2 that correspond to the Young's moduli of the PPy and the PVDF, respectively. The data needed to calculate the angle are obtained using the setup shown in Figure 4.

\section{A. Actuation Effect}

The mechanical, electrical and chemical properties of the actuator cannot be taken into account directly in establishing a mathematical model. Indeed, ANSYS cannot model the migration of ions or the electrostatic interactions between the polymer backbone and the ion. It is important to understand the distinction between the classical beam models and the model to be developed here. The classical models are valid when a passive beam is under a load. Here the beam is the actuator, which bends under its own ' electrochemomechanical' action. The actuation effect is actually produced by the volume change of the PPy layers (expansion or contraction). Hence the mathematical model must be based on a load generated as a result of the volume change. ANSYS can take into account some coupled effects, like thermal-structural effects. Therefore, an augmentation of the volume via a thermal expansion coefficient in a thermalstructural simulation comes to mind.

\section{MATHEMATICAL ANALYSIS}

In a classical structural analysis, the equations of the theory of elasticity are based on the Hooke's law expressed in 3D by Eq.1, where the strain $\varepsilon_{S}$ and the stress $\sigma$ in the body are linked by the deformation matrix [D].

$$
\begin{gathered}
\{\sigma\}=[D] \cdot\left\{\varepsilon_{S}\right\} \\
\text { where }\left\{\varepsilon_{S}\right\}=\left[\begin{array}{llllll}
\varepsilon_{x} & \varepsilon_{y} & \varepsilon_{z} & \gamma_{x y} & \gamma_{y z} & \gamma_{z x}
\end{array}\right]^{T} \text { and } \\
\{\sigma\}=\left[\begin{array}{llllll}
\sigma_{x} & \sigma_{y} & \sigma_{z} & \sigma_{x y} & \sigma_{y z} & \sigma_{z x}
\end{array}\right]^{T}
\end{gathered}
$$

Previous studies show that the strain can be related linearly to the applied voltage $\mathrm{V}[1]$, [6];

$$
\varepsilon_{S}=\beta . V
$$

The strain due to the expansion or contraction of the PPy under a voltage load can be considered as isotonic and without shearing. In order to take into account the interaction between the mechanical behaviour and the actuation principle, we can express the equation of the "electroelasticity' that occurs in the actuator as follows:

$$
\{\sigma\}=[D] \cdot\left(\left\{\varepsilon_{S}-\varepsilon_{V}\right\}\right)
$$

where $\left\{\varepsilon_{V}\right\}=\beta \cdot V \cdot\left[\begin{array}{llllll}1 & 1 & 1 & 0 & 0 & 0\end{array}\right]^{T}$

where ' $\mathrm{T}$ ' is the transpose of the vector. We use the FE software ANSYS to solve the mathematical model described by the Eq.3. ANSYS does not offer a module for this particular equation. Nevertheless, the software includes a 
coupled-field analysis based on the theory of thermoelasticity. This analysis takes into account the interaction between the mechanical and thermal fields and uses the following equation:

$$
\{\sigma\}=[D] \cdot\left(\left\{\varepsilon_{S}-\varepsilon_{T}\right\}\right)
$$

where $\left\{\varepsilon_{T}\right\}=\alpha \cdot T \cdot\left[\begin{array}{llllll}1 & 1 & 1 & 0 & 0 & 0\end{array}\right]^{T}$

where $T(x, y, z)$ is the non-uniform temperature field in the body and $\alpha$ is the coefficient of thermal expansion. In our study, we can use the thermo-elasticity equation if we mimic the voltage by the temperature $\mathrm{T}$, and the coefficient of thermal expansion by the slope $\beta$ of the strain-voltage linear approximation (Eq.2). That is;

$$
V \equiv T, \quad \beta \equiv \alpha
$$

The performance of the bending-type PPy actuator is quantified in terms of bending angle and bending moment. The finite element software (i) combines and solves the model equations, (ii) combines each element's strain equations in order to find the form of the structure made up of these elements, and in the same way, (iii) it combines all the forces produced by each element to determine the force produced by the structure made by these elements.

\section{ADJUSTING MODEL PARAMETERS}

The object of this section is to adjust a set of parameters resulting in a close correspondence between the experimental and theoretical bending angles and bending moments. These parameters are (i) the Young's moduli of the PPy and the PVDF, (ii) the coefficient of thermal expansion, and (iii) the temperature difference as the input parameter. It is important to notice that the mathematical model is a black-box model; the numerical values of the parameters do not necessarily represent their physical values and meanings. For instance, a load of $1 \mathrm{~V}$ gives, a strain of $0.3 \%$ which corresponds to a bending angle of $\theta=14.3$ degrees and a bending moment of $\mathrm{M}=0.18 \mathrm{mN} . \mathrm{cm}$. These parameters will be referred to $\theta_{\text {exp. }}$ and $\mathrm{M}_{\text {exp. }}$ A thermal load of $\Delta T=1^{\circ} \mathrm{F}$ with $\mathrm{E}_{\mathrm{PVDF}}=612 \mathrm{MPa}$, $\mathrm{E}_{\mathrm{PPy}}=80 \mathrm{MPa}$ (experimental values), $\boldsymbol{\alpha}=0.03$ do not produce the real $\theta_{\text {exp. }}$ and $\mathrm{M}_{\exp }$ values. This is due to the input/output based black-box nature of the mathematical model.

\section{A. Bending Angle ( $\theta)$}

As given before, the output displacement of the PPy actuator is evaluated in terms of the bending angle for the sake of making it independent of the actuator length. It is assumed that the bending angle of a PPy strip is directly proportional to the strain. Further, the bending angle $\theta$ depends on the strain of each element of the structure, and this strain can be obtained from Eq.4. Obviously, changing the coefficient of the thermal expansion $\boldsymbol{\alpha}$ will change the strain of each element and consequently, the bending angle of the strip.
Step 1 :

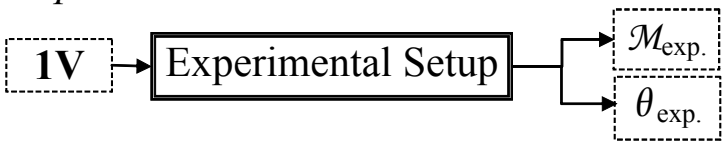

Step 2 :

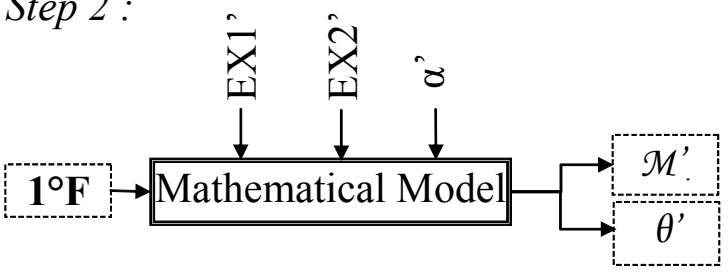

Step 3 :
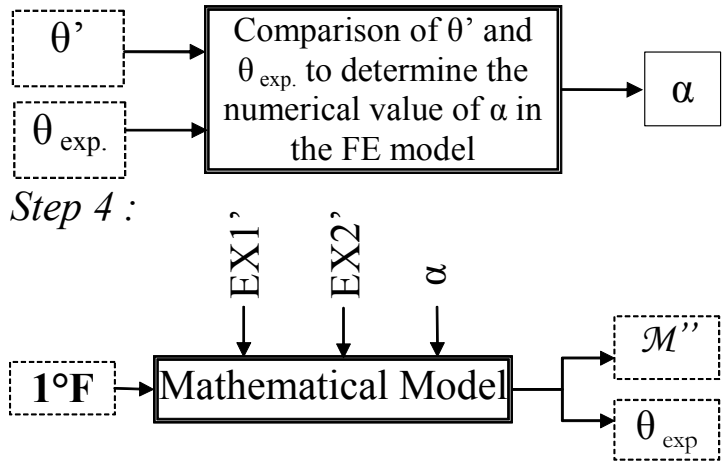

Step 5 :
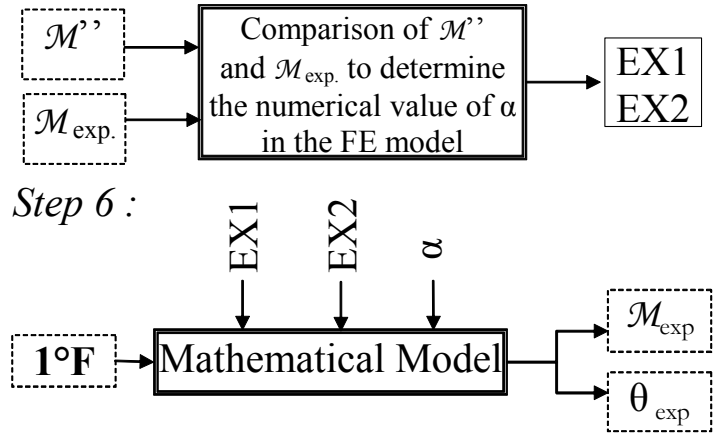

Figure 5: Adjustment of model parameters.

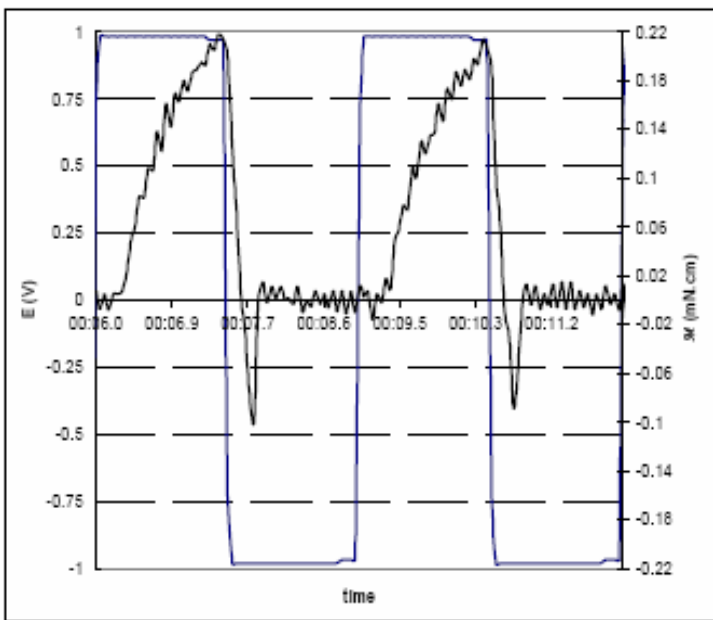

Figure 6: Bending moment experimental results $(-1 \mathrm{~V}, 1 \mathrm{~V}$ at $0.33 \mathrm{hz})$. 


\section{B. Bending Moment (M)}

As described by Eq. 4, the stress and the strain are linked by $\{\sigma\}=[D] \cdot\left(\left\{\varepsilon_{S}-\varepsilon_{V}\right\}\right)$ where $\sigma$ is the stress, $\varepsilon$ is the strain and $\mathrm{D}$ is proportional to the Young's modulus E. Based on Eq. 4, it is possible to calculate the value of the bending moment of the polypyrrole actuator by setting the Young modulus. Please note that we keep the value of the ratio EX1/EX2, but not the value of EX1 and EX2. Although each one will not have the same their true values, the mathematical model will take into account the stiffness of the PPy layer relative to the PVDF layer. The procedure to determine the parameters in the model is outlined in Figure 6. After determining the model parameters, the analogous model is ready to estimate the bending angle and bending moment outputs of the PPy bending actuator under different time-invariant voltages.

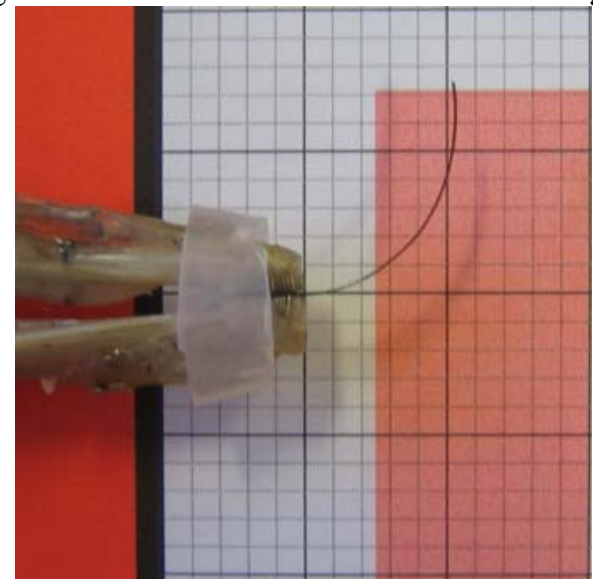

Figure 7: The configuration of the actuator while experimentally measuring its tip's vertical and horizontal displacements to calculate the bending angle $(-1 \mathrm{~V}, 1 \mathrm{~V}$ at $0.33 \mathrm{hz})$.

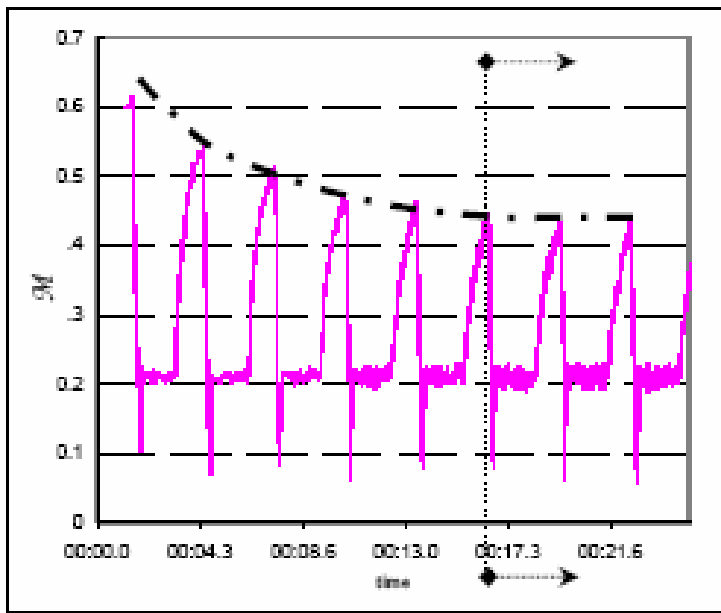

Figure 8: Reduction in the force output of the actuator after few cycles.

VI. MODEL VALIDATION BY EXPERIMENTATION

The input for the ANSYS model is a temperature difference that is analogous to the voltage applied to the PPy actuator. The experimental results given in Figure 6 and Figure 7 are for a PPy strip $10 \mathrm{~mm}$ in length, $1 \mathrm{~mm}$ in width and $12 \mathrm{~h}$ PPy grow with a current density of $1 \mathrm{~mA} / \mathrm{cm}^{2}$ (approximately 30 $\mu \mathrm{m}$ of PPy in each side of the $110 \mu \mathrm{m}$ PVDF layer). The experimental results are recorded after a number of cycles, due to the fact that the isotonic strain in the strip falls significantly during the 20 first cycles [2]. The force and displacement values are measured after the actuator response reaches its steady-state value, as illustrated in Figure 8.

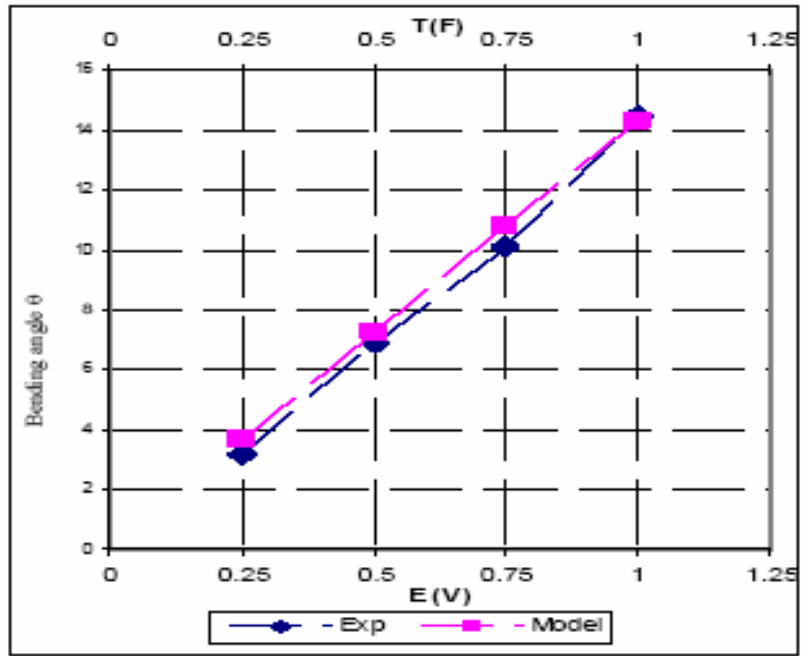

Figure 9: Experimental and simulated bending angle results.

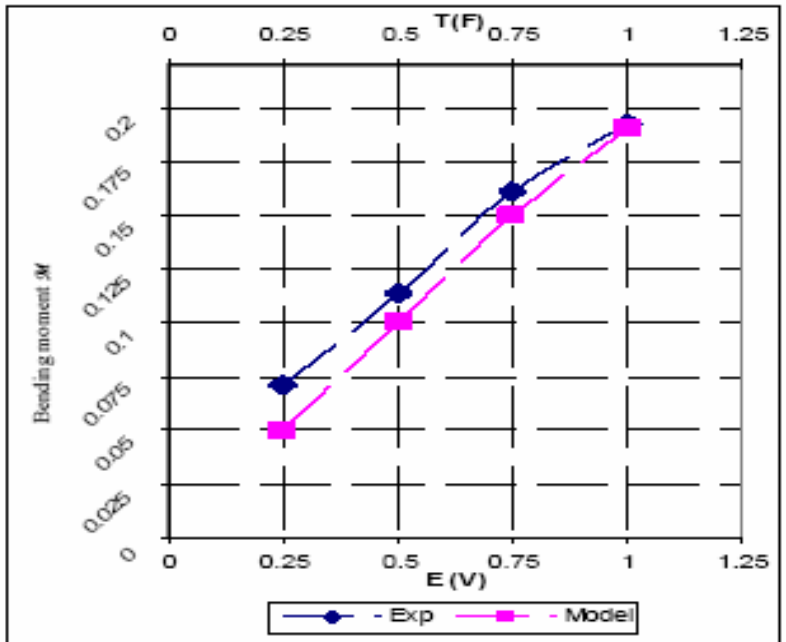

Figure 10: Experimental and simulated bending moment results.

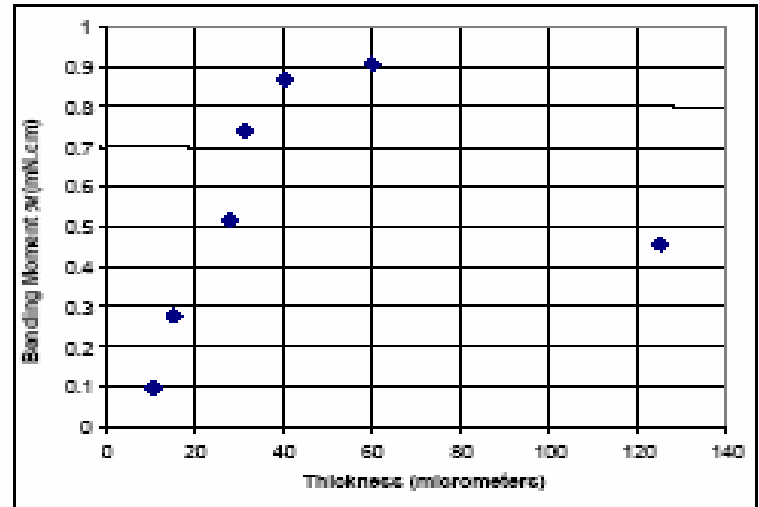

Figure 11: Experimental results showing the variation in the bending moment with the thickness. 
The experiments were run for $0.25 \mathrm{~V}, 0.5 \mathrm{~V}, 0.75 \mathrm{~V}$ and $1 \mathrm{~V}$. Many actuators were tested for each voltage to eliminate large inaccuracies in the experiments. The average values of the bending angle and the bending moment for each set of tests are shown in Figure 9 and Figure 10, respectively. Please note that the experimental results for $1 \mathrm{~V}$ are used to adjust the model parameters. The procedure described in Figure 6 has produced EX1 $=30.345 \mathrm{MPa}, \mathrm{EX} 2=232.143$ $\mathrm{MPa}$ and $\boldsymbol{\alpha}=0.01288$. With reference to Figure 9 and Figure 10 , the experimental and theoretical results are in a good agreement. This proves that the analogous temperature model is valid to predict the bending angle and bending moment of the PPy actuator for the range of $[-1 \mathrm{~V} ; 1 \mathrm{~V}]$.

\section{MODEL IMPROVEMENT}

The experimental bending moment output of the PPy actuator has been evaluated for different PPy thicknesses, and is provided in Figure 11, where there is a non-negligible drop in the bending moment when each of the PPy layers is thicker than $60 \mu \mathrm{m}$. The model established and validated in the previous section assumes that the actuation ability of the PPy layers is constant along the thickness. This means that the outer surfaces of the PPy layers are as effective as the inner surfaces in contact with PVDF layer. In reality, the ' electrochemomechanical' reaction is more effective around the vicinity of the voltage application - the fixed end of the actuator. This behaviour is mainly due to the migration of the moving ions into the PPy layers. The moving ions are relatively large and it is difficult for the PPy layer to be uniformly doped. The migration of ions starts close to the voltage application zone and diffuse to the depth of the PPy layer. This behaviour is complex to include in a fully analytical model. In this section, we improve the lumpedparameter model such that it takes into account the decrease in actuation performance with the distance from the voltage application point in order to quantify the real behaviour of the actuator for different thicknesses. We propose to use a gradient of the actuation efficiency in the PPy layer. The PPy close to the PVDF layer is more dominant than the other sections further from the PVDF layer, as schematically described in Figure 12.

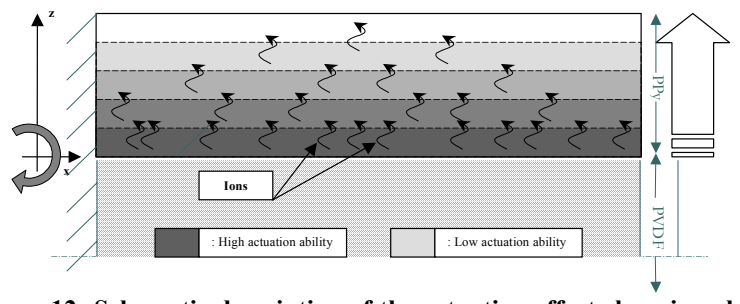

Figure 12: Schematic description of the actuation effect changing along the thickness of the PPy layers.

A set of simulations have been run with different actuation gradients to determine the behaviour of the mathematical model such that the model describes the variation of the bending moment and bending angle with the PPy thickness. Comparison of the simulation results with experimental ones has proven that including the actuation gradient in the model improves the ability of the model to predict the influence of the PPy layers' thickness on the bending angle and moment outputs of the actuator. Because of lack of space, the results could not be presented in this paper.

\section{CONCLUSIONS}

We have established a lumped-parameter mathematical model of a PPy actuator based on a finite element analysis. The model describes the input/output behaviour rather than the physical/chemical phenomenon behind the actuation. The model contains the volume change, the elastic deformation, the input voltage and the actuation ability gradient along the PPy thickness. This model is effective enough to predict the bending angle and bending moment outputs of the PPy actuators quite well for a range of input voltages. The mathematical model has been validated in the voltage domain and in the thickness domain such that it can be used to optimise the geometry of PPy layers for different applied voltages.

\section{ACKNOWLEDGEMENTS}

This project has been funded in part by a URC Small Grant. The authors wish to express their gratitude for the assistance provided by the Intelligent Polymer Research Institute at the University of Wollongong.

\section{REFERENCES}

1. G. Alici, P. Metz, and G. M. Spinks, "Size optimisation of polypynrole (PPy) actuators for micro/nano manipulation systems", 2005 IEEE Int. Conference on Robotics and Biomimetics, Hong Kong, June 2005.

2. G. M. Spinks, B. Xi, D. Zhou, V. T. Truong, and G. G. Wallace, "Enhanced control and stability of polypynrole electromechanical actuators", Synthetic Metals, Vol.140, pp. 273 - 280, 2004.

3. E. Smela, "Conjugated Polymer Actuators for Biomedical Applications", Advanced Materials, Vol.15, No.6, pp. 481 - 494, March 2003.

4. J. W. L. Zhou, H. Y. Chan, T. K. H. To, K. W. C. Lai, and W. L. Li, "Polymer MEMS actuators for underwater micromanipulation", IEEE/ASME Trans, on Mechatronics, Vol.9, No.2, pp. $334-342$, 2004.

5. E. W. H. Jager, E. Smela, O. Inganas, and I. Lundstrom, "Polypynrole Microactuators", Synthetic Metals, pp. $1309-1310,1999$.

6. E. Smela, M. Kallenbach, and Jens Holdenried, "Electrochemically Driven polypynrole bilayers for moving and positioning bulk micromachined silicon plates", IEEE Joumal of Microelectromechanical Systems, Vol.8, No.4, pp. 373 - 383, December 1999.

7. R. H. Baughman, "Conducting polymer artificial muscles," Synthetic Metals, vol. 78, pp. 339-353, 1996.

8. G. M. Spinks, L. Liu, G. G. Wallace, D. Zhou, "Strain response from polypyrrole actuators under load," Advance Functional Materials, vol. 12 , no. 6-7, pp. 437-440, 2002.

9. J.D. Madden, R.A.Cush, T.S.Kanigan, and I.W. Hunter, "Fast contracting polypyrrole actuators", Synthetic Metals, 113, pp. 185 192,2000

10. A. Della Santa, D. De Rossi, A. Mazzoldi, "Performance and work capacity of a polypyrrole conducting polymer linear actuator", Synthetic Metals, vol. 90, pp. 93-100, 1997

11. A. Della Santa, D. De Rossi, A. Mazzoldi, "Characterisation and modelling of a conducting polymer muscle-like linear actuator", Smart Materials and Structures, vol. 6, pp. 23-34, 1997.

12. S. Nemat-Nasser, J.Y. Li, "Electromechnanical response of ionic polymer-metal composites," J. Applied Physics, vol. 87, no. 7, pp. $3321-3331,2000$

13. S. Nemat-Nasser, "Micro-mechanics of Actuation of Ionic Polymermetal Composites," J. A. Physics, vol. 92, no. 5, pp. 2899-2915, 2002.

14. Lobo H., Bonilla J. V., Handbook of Plastics analysis, Marcel Dekker, Inc, New York. 2003.

15. Elias H-G. An Introduction to Plastics, Germany : Wiley-VCH, 2003.

16. Ward I. M., Hadley D. W., An introduction to the Mechanical Properties of Solid Polymers, Chichester, John Wiley \&Sons Ltd, 1993. 